\title{
Reliability Model Development for Wind Turbine Drivetrain with Brushless Doubly-Fed Induction Machine as Generator
}

\author{
Salman Abdi \\ School of Engineering, \\ University of East Anglia (UEA) \\ Norwich, UK \\ S.abdi-jalebi@uea.ac.uk
}

\author{
Sara Sharifzadeh \\ School of Computing, Electronics and \\ Mathematics, Coventry University \\ Coventry, UK \\ Ac8115@ coventry.ac.uk
}

\author{
Sam Amiri \\ School of Computing, Electronics and \\ Mathematics, Coventry University \\ Coventry, UK \\ Ad0246@coventry.ac.uk
}

\begin{abstract}
Brushless doubly-fed induction machines (BDFIM) are attractive generators to be used in wind turbines due to the absence of brushes and slip rings. Furthermore, the BDFIM is a medium-speed generator and hence only requires one or two-stage gearbox. This feature simplifies the gearbox system and therefore improve reliability and reduce maintenance costs for the wind turbine. Although the design and operation of the BDFIM has been widely studied in the literature, there are only few studies on reliability assessment of the machine as a wind turbine generator. This paper proposes a comprehensive reliability model for two wind turbine drivetrain configurations: One with doubly-fed induction generator, and the other when the BDFIM is employed as the generator. The model is capable of evaluating the failure rate and repair rate indexes for the both configurations. Real field survey data from a $90 \mathrm{MW}$ wind farm as well as calculated reliability data are then utilised to determine the reliability index values for the two drivetrain configurations in order to compare their reliability performance.
\end{abstract}

Keywords - brushless Doubly-fed induction machines, failure rate, mean time to failure, mean time to repair, reliability assessment, repair rate, wind turbine drivetrain.

\section{INTRODUCTION}

As wind turbines penetration continues to grow, the effect of wind turbine reliability is becoming an important consideration. The competitive power generation industry means that the developers and operators seek the most economically productive wind turbine configuration. Long-term reliability and cost analysis of wind turbines, including their capital and operational and maintenance $(\mathrm{O} \& \mathrm{M})$ costs will result in the selection of the most appropriate drivetrain configuration. This is only possible if such analysis includes the reliability of different wind turbine technologies.

There has been a dramatic increase in the size of wind turbines from $30 \mathrm{~kW}$ up to $10 \mathrm{MW}$ Over the past 50 years [1]. To date, several generator concepts have been considered for offshore wind where there is substantial wind power resource and hence significant future market growth is expected. These include permanent magnet generators, doubly fed induction generators (DFIG), superconducting generators and high voltage DC generators [2]. To reduce the cost of energy from wind turbines, there is a need to improve availability and reduce operational and maintenance costs.
The brushless doubly fed induction machine (BDFIM) is an attractive solution as a wind power generator particularly for the wind turbines erected offshore. The BDFIM retains the benefits of using fractional-sized converter as with the DFIG used in the majority of wind turbines. Nevertheless, the brushless operation and slip-ring elimination in a BDFIM can enhance the generator reliability significantly compared to the DFIG generator [3]. In addition, BDFIM is a medium-speed machine and hence it only requires a one or two-stage gearbox system, compared to the complex 3-stage gearbox system needed for the DFIG drivetrain configuration. This can bring further reliability improvement for the overall drivetrain [4].

Although the above advantages are stated for the BDFIM over the widely used DFIG, there is only little work carried out to study and quantify the BDFIM's reliability benefits and maintenance cost reductions for the wind turbine operators. The only study on the BDFIM reliability, to the best of the authors' knowledge, was presented in [5]. In this study, failure mode and effect analysis (FMEA) method was used to determine risk priority number (RPN) index for different subassemblies of a 2 MW wind turbine drivetrain for the two cases of when DFIG and BDFIM are presented as generator. It was shown that the overall drivetrain's reliability can be improved significantly when a high-speed DFIG is replaced by a medium-speed BDFIM.

There are also a few studies carried out on the fault diagnosis of the BDFIM. Afshar et. al. in [6] proposed a fault detection method to detect rotor eccentricity faults in the BDFIM by identifying the signature frequencies in the stator current signals. They also proposed in [7] a diagnosis method to detect the stator inter-turn short circuit faults in the stator windings using a continuous wavelet transform method. Logan et. al. [8] studied the vibration patterns in the BDFIM's main mode of operation i.e. synchronous mode, and derived equations for vibration components magnitudes generated by the machine's complex magnetic fields as functions of air-gap flux density, pole numbers and machine dimensions. Abdi et. al. in [9] proposed a new parallel winding design for the stator PW and CW to mitigate the vibration level in the presence of rotor eccentricity.

This paper aims at developing a generic reliability model for the main systems of the wind turbine drivetrain including the generator, power converter and gearbox. These systems have 
been shown to be the most critical drivetrain subassemblies in a wind turbine [10]. The model proposes two distinct reliability formulations for a high-speed DFIG and a medium-speed BDFIM drivetrain configurations. The proposed model is then utilised to compare the reliability of those drivetrain configurations using field survey reliability data obtained from a $90 \mathrm{MW}$ wind farm as well as analytical reliability data where the real data were not existed.

\section{BRUSHLESS DOUBLY-FED INDUCTION MACHINE}

The brushless doubly-fed induction machine is a variable speed electrical machine with two sets of three-phase windings in a common stator frame. As a generator it is particularly attractive for wind power generation where one stator winding is directly connected to the grid, called the power winding (PW), and the other stator winding is supplied by a variable-voltage variable-frequency converter, called the control winding (CW) [11]. Fig. 1 shows the BDFIM grid connection as a wind generator. The desirable mode of operation for the BDFIM is the synchronous mode where both stator windings are supplied. In this mode of operation, the rotor speed is controlled by controlling the CW supplied frequency using a fractional-sized power converter. The rotor speed can be obtained from [12]:

$$
\omega_{r}=\frac{\omega_{1}+\omega_{2}}{p_{1}+p_{2}}
$$

where $p_{1}$ and $p_{2}$ are the number of pole pairs, and $\omega_{1}$ and $\omega_{2}$ are the supplied frequencies for PW and CW, respectively.

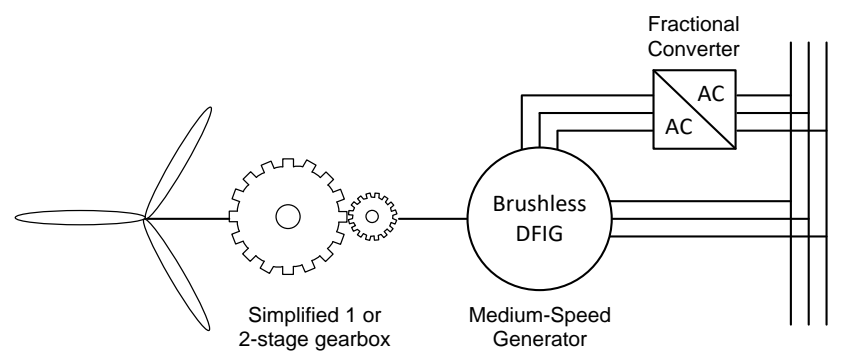

Fig. 1. A schematic of the BDFIM drivetrain configuration

The stator windings' pole pair numbers are chosen in a way to avoid direct coupling between the windings [11]. The coupling is enabled using a special design of the rotor, a nestedloop design has shown robust operation and acceptable performance [3]. The number of nests for the rotor needed for cross coupling of PW and CW can be obtained from:

$$
N_{n}=p_{1}+p_{2}
$$

Several attempts have been reported to design and construct large-scale prototype BDFIMs for example, in Brazil with a 75 $\mathrm{kW}$ machine [13], in China with a $200 \mathrm{~kW}$ machine [14] and in the UK with $250 \mathrm{~kW}$ machine [15], the later was built and tested successfully by the authors and as a stepping-stone towards MW-scale wind turbine generator.

Although several aspects of the BDFIM design and performance have been studied previously, for instance the weight and size optimisation [16], vibration analysis [11], grid synchronisation [17] and low-voltage ride-through (LVRT) capabilities [15], there are only a few studies reported on the reliability assessment of the generator. This is particularly important to evaluate and quantify the advantages of the BDFIM technology as a wind turbine generator over its well-known counterpart i.e. DFIG used in the majority of wind turbines before designing and constructing MW-scale machines.

\section{WIND TURBINE DRIVETRAIN RELIABILITY MODEL}

Failure rate $(\lambda)$ and repair rate $(\mu)$ are the two well-known indexes employed for reliability analysis of wind turbines [18]. Failure rate is defined as the number of failures occurred in a given time period divided by the total time the component was in operation. Repair rate is defined as the number of repairs carried out in a given time period divided by the total time the component was being repaired. The failure and repair rates can be calculated as:

$$
\begin{aligned}
& \lambda=\frac{1}{M T T F} \\
& \mu=\frac{1}{M T T R}
\end{aligned}
$$

where MTTF is the mean time to failure and MTTR is the mean time to repair. Recording the period the wind turbine was in operation and repair, as well as taking the average of uptime and downtime gives numerical values for MTTF and MTTR, respectively. The same procedure can be conducted for each subassembly to calculate the corresponding failure and repair rates. The drivetrain is first broken down into its subassemblies. After this division, a reliability model of each subassembly can be derived and a system reliability model can be constructed by combining the individual subassembly reliability models. This paper will focus on generator, power converter, and gearbox reliability data of wind turbines gathered in a three-year survey conducted in a $90 \mathrm{MW}$ wind farm in Iran as the main modes of failures and wind turbine downtime [19].

\section{A. Generator Reliability Model}

An induction-typed generator such as a DFIG is a system consisting of several subassemblies, namely the stator and rotor windings, the magnetic core, the shaft and bearings and terminal boxes. Only the generator subassemblies shown in Table I are considered in this study with highest failure rates among other generator subassemblies.

\section{TABLE I. GENERATOR RELIABILITY MODEL PARAMETERS}

\begin{tabular}{ll}
\hline Parameter & Description \\
$\lambda_{\mathrm{s}(\mathrm{r})}$ & Failure rate of stator (rotor) winding \\
$\mu_{\mathrm{s}(\mathrm{r})}$ & Repair rate of stator (rotor) winding \\
$\lambda_{\mathrm{bs}}$ & Failure rate of generator bearings and shaft \\
$\mu_{\mathrm{bs}}$ & Repair rate of generator bearings and shaft \\
$\lambda_{\mathrm{br}}$ & Failure rate of brushes and slip rings \\
$\mu_{\mathrm{br}}$ & Repair rate of brushes and slip rings \\
\hline
\end{tabular}

Since problems associated with either the stator or rotor winding will result in a generator fault, these two subassemblies are considered in series from a reliability point of view, so their 
failure and repair rates can be combined to form a generator winding assembly, denoted by the subscript "gen.wind":

$$
\begin{gathered}
\lambda_{\text {gen.wind }}=\lambda_{s}+\lambda_{r} \\
\mu_{\text {gen.wind }}=\frac{\mu_{s} \mu_{r}\left(\lambda_{s}+\lambda_{r}\right)}{\mu_{s} \lambda_{r}+\mu_{r} \lambda_{s}}
\end{gathered}
$$

Similarly, because brushes and slip rings (denoted by the subscript "br") as well as bearing and shaft (denoted by the subscript "bs") failures can result in a generator fault, these two subassemblies are considered in series and denoted by the subscript "gen.mech":

$$
\begin{gathered}
\lambda_{\text {gen.mech }}=\lambda_{b s}+\lambda_{b r} \\
\mu_{\text {gen.mech }}=\frac{\mu_{b s} \mu_{b r}\left(\lambda_{b s}+\lambda_{b r}\right)}{\mu_{b s} \lambda_{b r}+\mu_{b r} \lambda_{b s}}
\end{gathered}
$$

Finally, combining the "gen.wind" and "gen.mech" subassemblies leads to a reduced DFIG reliability model:

$$
\begin{gathered}
\lambda_{\text {dfig }}=\lambda_{\text {gen.wind }}+\lambda_{\text {gen.mech }} \\
\mu_{\text {dfig }}=\frac{\mu_{\text {gen.wind }} \mu_{\text {gen.mech }}\left(\lambda_{\text {gen.wind }}+\lambda_{\text {gen.mech }}\right)}{\mu_{\text {gen.wind }} \lambda_{\text {gen.mech }}+\mu_{\text {gen.mech }} \lambda_{\text {gen.wind }}}
\end{gathered}
$$

A BDFIM reliability model is based on the DFIG model, however, with the absence of the brushes and slip rings "br". The resulting reliability model is:

$$
\begin{gathered}
\lambda_{b d f i m}=\lambda_{\text {gen.wind }}+\lambda_{b s} \\
\mu_{b d f i m}=\frac{\mu_{\text {gen.wind }} \mu_{b s}\left(\lambda_{\text {gen.wind }}+\lambda_{b s}\right)}{\mu_{\text {gen.wind }} \lambda_{b s}+\mu_{b s} \lambda_{\text {gen.wind }}}
\end{gathered}
$$

\section{B. Converter Reliability Model}

The power electronic converter consists of two inverters: the machine-side (rotor-side for DFIG configuration) and grid-side inverter connected back-to-back with a DC-link capacitor between the two inverters as shown on Fig. 2. A controller is used to control the output voltage and frequency of the machineside inverter, thus controlling the speed and power factor of the generator, and to maintain a constant DC-link voltage by controlling the grid side inverter. The converter assessed in this paper is made up of the subassemblies shown in Table II.

Machine and grid-side inverter faults have similar effects on converter failure. However, their failure and repair rates are considered separately. The combined effects of the two subassemblies are denoted with the subscript "pe", indicating power electronics.

$$
\begin{gathered}
\lambda_{p e}=\lambda_{m . i n v}+\lambda_{g . i n v} \\
\mu_{p e}=\frac{\mu_{m . i n v} \mu_{g . i n v}\left(\lambda_{m . i n v}+\lambda_{g . i n v}\right)}{\mu_{m . i n v} \lambda_{g . i n v}+\mu_{g . i n v} \lambda_{m . i n v}}
\end{gathered}
$$

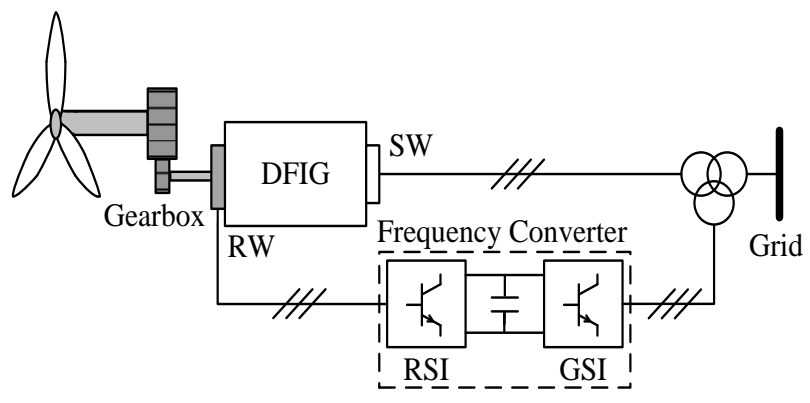

(a)

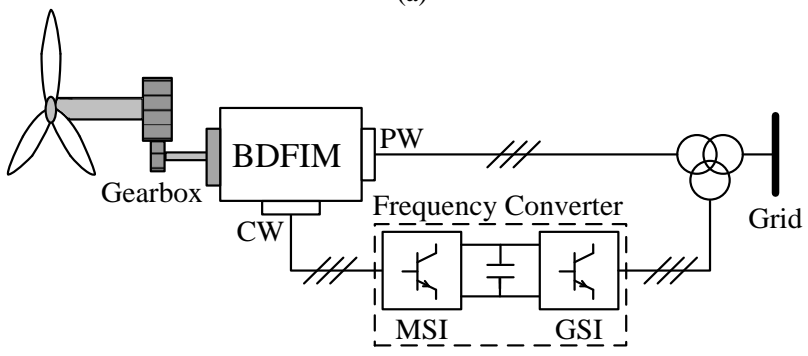

(b)

Fig. 2. DFIG and BDFIM drivetrain configurations

TABLE II. CONVERTER RELIABILITY MODEL PARAMETERS

\begin{tabular}{ll}
\hline Parameter & Description \\
$\lambda_{\text {m.inv(g.inv) }}$ & Failure rate of machine (grid) side inverter \\
$\mu_{\text {m.nnv(g.nv) }}$ & Repair rate of machine (grid) side inverter \\
$\lambda_{\mathrm{cu}}$ & Failure rate of control unit \\
$\mu_{\mathrm{cu}}$ & Repair rate of control unit \\
$\lambda_{\mathrm{dc}}$ & Failure rate of DC-link \\
$\mu_{\mathrm{dc}}$ & Repair rate of DC-link \\
\hline
\end{tabular}

The DC-link and control unit component failures result in converter malfunction, so these two components can be considered as a series system in converter reliability assessment, and equivalent parameters of this combined subassembly "dcu" are:

$$
\begin{gathered}
\lambda_{d c u}=\lambda_{d c}+\lambda_{c u} \\
\mu_{d c u}=\frac{\mu_{d c} \mu_{c u}\left(\lambda_{d c}+\lambda_{c u}\right)}{\mu_{d c} \lambda_{c u}+\mu_{c u} \lambda_{d c}}
\end{gathered}
$$

Finally, combining the "dcu" and "pe" subassemblies, the same procedure used for the generator reliability model, leads to a reduced converter reliability model:

$$
\begin{gathered}
\lambda_{\text {con }}=\lambda_{d c u}+\lambda_{p e} \\
\mu_{c o n}=\frac{\mu_{d c u} \mu_{p e}\left(\lambda_{d c u}+\lambda_{p e}\right)}{\mu_{d c u} \lambda_{p e}+\mu_{p e} \lambda_{d c u}}
\end{gathered}
$$

\section{Gearbox Reliability Model}




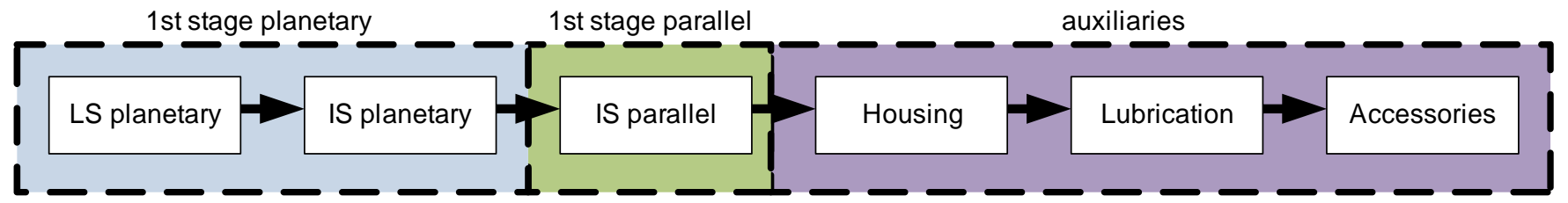

(a)

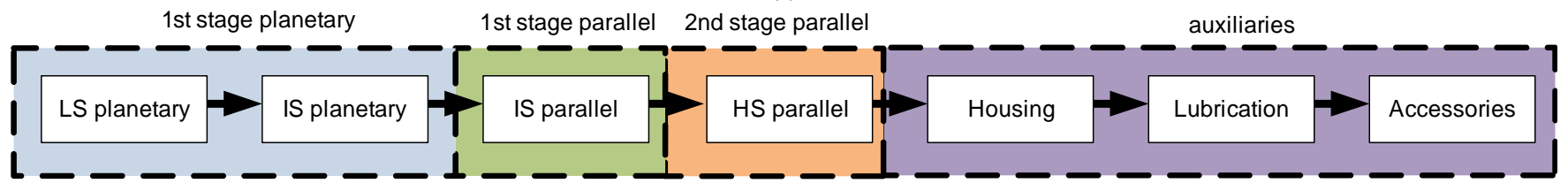

(b)

Fig. 3. Block diagrams of a) 2-stage, b) 3-stage gearbox configurations for wind turbine drivetrain.

In [20], the reliability of wind turbine gearboxes is analysed. Two gearbox configurations are presented: A 3-stage gearbox comprising of two planetary stages followed by one parallel stage with a transmission ratio of approximately 126 , and a 2stage gearbox comprised of one planetary stage followed by one parallel stage with a transmission ratio of approximately 35 . The block diagrams of different gearbox assemblies are shown in Fig. 3. The gearbox reliability model parameters are shown in Table III.

TABLE III. GeARBox RELIABILITY MODEL PARAMETERS

\begin{tabular}{ll}
\hline Parameter & Description \\
$\lambda_{\mathrm{l}(\mathrm{i}) \mathrm{p}}$ & Failure rate of low (intermediate) speed planetary gear \\
$\mu_{\mathrm{l( \textrm {i } ) \mathrm { p }}}$ & Repair rate of low (intermediate) speed planetary gear \\
$\lambda_{\mathrm{i}(\mathrm{h}) \mathrm{pa}}$ & $\begin{array}{l}\text { Failure rate of intermediate (high) speed parallel stage } \\
\text { gear }\end{array}$ \\
$\mu_{\mathrm{i}(\mathrm{h}) \mathrm{pa}}$ & $\begin{array}{l}\text { Repair rate of intermediate (high) speed parallel stage } \\
\lambda_{\mathrm{h}}\end{array}$ \\
$\mu_{\mathrm{h}}$ & Feailure rate of gearbox housing \\
$\lambda_{\mathrm{l}}$ & Repair rate of gearbox housing \\
$\mu_{\mathrm{l}}$ & Failure rate of lubrication system \\
$\lambda_{\mathrm{ax}}$ & Repair rate of lubrication system \\
$\mu_{\mathrm{ax}}$ & Failure rate of gearbox accessories \\
\hline
\end{tabular}

a) 2-stage Gearbox: Failure of either planetary (high and intermediate speed side) or parallel gear will result in a gearbox failure and as such these subsystems can be considered a series system resulting in the "2g.gears" subassembly:

$$
\begin{gathered}
\lambda_{2 \text { g.gears }}=\lambda_{l p}+\lambda_{i p}+\lambda_{\text {ipa }} \\
\mu_{2 \text { g.gears }}=\frac{\mu_{l p} \mu_{i p} \mu_{i p a}\left(\lambda_{l p}+\lambda_{i p}+\lambda_{i p a}\right)}{\mu_{l p} \mu_{i p} \lambda_{i p a}+\mu_{l p} \mu_{i p a} \lambda_{i p}+\mu_{i p} \mu_{i p a} \lambda_{l p}}
\end{gathered}
$$

Similarly, the housing, lubrication and accessories can be considered in series, resulting in the " $2 \mathrm{~g}$. aux" subassembly.

$$
\lambda_{2 \text { g.aux }}=\lambda_{h}+\lambda_{l}+\lambda_{a x}
$$

$$
\mu_{2 \text { g.aux }}=\frac{\mu_{h} \mu_{l} \mu_{a x}\left(\lambda_{h}+\lambda_{l}+\lambda_{a x}\right)}{\mu_{h} \mu_{l} \lambda_{a x}+\mu_{h} \mu_{a x} \lambda_{l}+\mu_{l} \mu_{a x} \lambda_{h}}
$$

Combining the gears and auxiliary subsystems of the 2-stage gearbox together results in the reduced 2-stage gearbox reliability model " $2 \mathrm{~g}$ ":

$$
\lambda_{2 g}=\lambda_{2 \text { g.gears }}+\lambda_{2 g . a u x}
$$

$$
\mu_{2 g}=\frac{\mu_{2 \text { g.gears }} \mu_{2 \text { g.aux }}\left(\lambda_{2 \text { g.gears }}+\lambda_{2 \text { g.aux }}\right)}{\mu_{2 \text { g.gears }} \lambda_{2 \text { g.aux }}+\mu_{2 \text { g.aux }} \lambda_{2 \text { g.gears }}}
$$

b) 3-stage Gearbox: The 3-stage gearbox reliability model is developed by modifying the reliability model of the 2stage gearbox by including an additional parallel gear stage, resulting in the "3g.gears" subsystem.

$$
\begin{gathered}
\lambda_{3 \text { g.gears }}=\lambda_{2 \text { g.gears }}+\lambda_{\text {hpa }} \\
\mu_{3 \text { g.gears }}=\frac{\mu_{2 \text { g.gears }} \mu_{h p a}\left(\lambda_{2 \text { g.gears }}+\lambda_{h p a}\right)}{\mu_{2 \text { g.gears }} \lambda_{h p a}+\mu_{h p a} \lambda_{2 \text { g.gears }}}
\end{gathered}
$$

The "3g.aux" subsystem is comprised of the same elements as the "2g.aux" subsystem.

$$
\begin{aligned}
& \lambda_{3 \text { g.aux }}=\lambda_{2 \text { g.aux }} \\
& \mu_{3 \text { g.aux }}=\mu_{2 \text { g.aux }}
\end{aligned}
$$

Combining the gears and auxiliary subsystems of the 3 -stage gearbox together results in the reduced 3-stage gearbox reliability model " $3 \mathrm{~g}$ ":

$$
\begin{gathered}
\lambda_{3 g}=\lambda_{3 \text { g.gears }}+\lambda_{3 \text { g.aux }} \\
\mu_{3 g}=\frac{\mu_{3 \text { g.gears }} \mu_{3 \text { g.aux }}\left(\lambda_{3 \text { g.gears }}+\lambda_{3 \text { g.aux }}\right)}{\mu_{3 \text { g.gears }} \lambda_{3 \text { g.aux }}+\mu_{3 \text { g.aux }} \lambda_{3 \text { g.gears }}}
\end{gathered}
$$

\section{Complete Drivetrain Reliability Model}

From (5) to (30), the reliability indexes for the drivetrain with DFIG generator, 3-stage gearbox and partially rated converter (with subscript "dfig.dt") can be expressed as: 


$$
\begin{gathered}
\lambda_{\text {dfig.dt }}=\lambda_{\text {dfig }}+\lambda_{\text {con }}+\lambda_{3 g} \\
\mu_{\text {dfig.dt }} \\
=\frac{\mu_{d f i g} \mu_{c o n} \mu_{3 g}\left(\lambda_{d f i g}+\lambda_{c o n}+\lambda_{3 g}\right)}{\mu_{d f i g} \mu_{c o n} \lambda_{3 g}+\mu_{d f i g} \mu_{3 g} \lambda_{c o n}+\mu_{3 g} \mu_{c o n} \lambda_{d f i g}}
\end{gathered}
$$

In addition, the reliability indexes for the drivetrain with BDFIM generator, 2-stage gearbox and partially rated converter (with subscript "bdfim.dt") can be expressed as:

$$
\lambda_{\text {bdfim.dt }}=\lambda_{\text {bdfim }}+\lambda_{\text {con }}+\lambda_{2 g}
$$

$$
\begin{aligned}
& \mu_{\text {bdfim.dt }} \\
& =\frac{\mu_{\text {bdfim }} \mu_{\text {con }} \mu_{2 g}\left(\lambda_{\text {bdfim }}+\lambda_{\text {con }}+\lambda_{2 g}\right)}{\mu_{\text {bdfim }} \mu_{\text {con }} \lambda_{2 g}+\mu_{b d f i m} \mu_{2 g} \lambda_{\text {con }}+\mu_{2 g} \mu_{\text {con }} \lambda_{b d f i m}}
\end{aligned}
$$

\section{EXPERIMENTAL EVALUATION}

Manjil is a $90 \mathrm{MW}$ wind farm located in northern Iran in a mountainous environment near Manjil city (Fig. 4) consisting of 72 fixed and variable-speed geared wind turbines incorporating induction generators manufactured by NEG, Vestas and NordTank. The site has high wind speeds, a dusty and humid climate, competent maintenance staff, easy access and a repair facility. The reliability data for generator and converter published in [19] is shown in Table IV, the data was gathered over a 3-year period where the failure types, root causes and time lost to repair were documented by maintenance staff. The time lost to repair gives an approximation of the repair time (MTTR) for each failure modes for this wind farm's conditions, including availability of

\begin{tabular}{|c|c|c|c|}
\hline Assembly & Subassembly & $\begin{array}{l}\text { Failure rate } \\
\left(\text { year }^{-1}\right)\end{array}$ & $\begin{array}{l}\text { Repair rate } \\
\left(\text { year }^{-1}\right)\end{array}$ \\
\hline \multirow[t]{4}{*}{ Generator } & Stator & 0.02 & 36.5 \\
\hline & Rotor & 0.02 & 36.5 \\
\hline & Bearings & 0.08 & 60 \\
\hline & Brush gear & 0.1 & 243.3 \\
\hline \multirow[t]{4}{*}{ Converter } & MSI & $0.4\left(0.3^{*}\right)$ & 73 \\
\hline & GSI & $0.4\left(0.3^{*}\right)$ & 73 \\
\hline & Control unit & 0.01 & 4380 \\
\hline & DC link & 0.04 & 73 \\
\hline
\end{tabular}
spare parts and weather conditions. The failure rates of the machine and grid side inverters for a partially rated converter is 0.3 failures per year denoted by '*'.

TABLE IV. GeARbox Reliability Model PARAMETERS

Wind turbine gearbox reliability data is based on data gathered in [20]. The MTTR data is defined as the actual time to repair or replace a component. This implies that logistic delays and transportation times are excluded from the repair time. The values have been adjusted to attempt to account for logistic and transportation delays which would typically be experienced on a wind farm and to make the data more comparable to the generator and converter failure rate data shown in Table IV. The predicted failure rates of individual modules of different gearbox configurations, namely two- and three-stage gearboxes are given in Table $\mathrm{V}$. The values are derived from mathematical reliability predications using industrial formulae and assumptions. The data does not originate from field failure data of actual wind turbines.

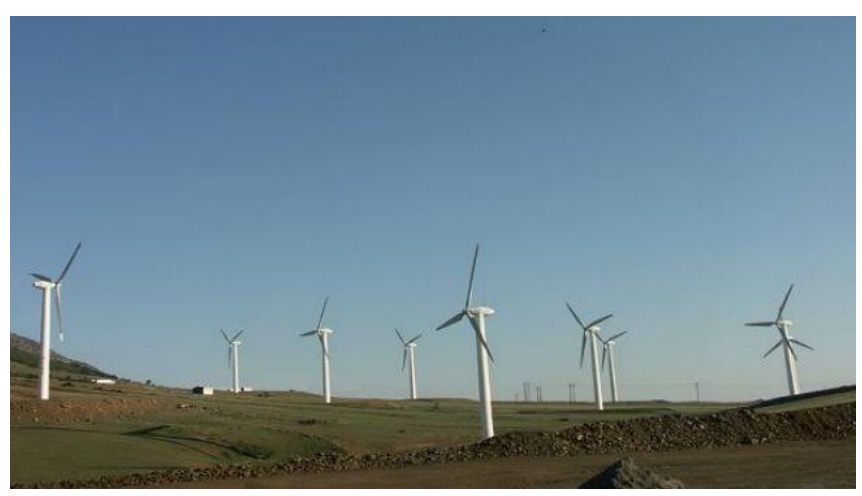

Fig. 4. $90 \mathrm{MW}$ wind farm located in north of Iran

TABLE V. Wind TURBINE GEARbox SUbASSEMBly RELIABILITy DATA

\begin{tabular}{llll}
\hline Assembly & Subassembly & $\begin{array}{l}\text { Failure rate } \\
\left(\text { year }^{-1}\right)\end{array}$ & $\begin{array}{l}\text { Repair rate } \\
\left(\text { year }^{-1}\right)\end{array}$ \\
\hline 2-stage & LS planetary & 0.012 & 30.4 \\
& IS planetary & 0.019 & 30.4 \\
& IS parallel & 0.009 & 60.8 \\
& Housing & 0.005 & 30.4 \\
& Lubrication & 0.018 & 91.3 \\
& Accessories & 0.005 & 365 \\
\hline 3-stage & LS planetary & 0.012 & 30.4 \\
& IS planetary & 0.019 & 30.4 \\
& IS parallel & 0.010 & 60.8 \\
& HS parallel & 0.027 & 60.8 \\
& Housing & 0.005 & 30.4 \\
& Lubrication & 0.018 & 91.3 \\
& Accessories & 0.005 & 365 \\
\hline
\end{tabular}

TABLE VI. CALCUlated Reliability MOdEL PARAMETERS

\begin{tabular}{llll}
\hline Failure rate & year $^{-1}$ & Repair rate & year $^{-1}$ \\
\hline$\lambda_{\text {dfig }}$ & 0.22 & $\mu_{\text {dfig }}$ & 77.5 \\
$\lambda_{\text {bdfim }}$ & 0.12 & $\mu_{\text {bdfim }}$ & 49.4 \\
$\lambda_{\text {con }}$ & $0.85(0.65)$ & $\mu_{\text {con }}$ & $73.9(74.1)$ \\
$\lambda_{3 \mathrm{~g}}$ & 0.10 & $\mu_{3 \mathrm{~g}}$ & 47.9 \\
$\lambda_{2 \mathrm{~g}}$ & 0.07 & $\mu_{2 \mathrm{~g}}$ & 44.1 \\
$\lambda_{\text {dfig.dt }}$ & 0.97 & $\mu_{\text {dfig.dt }}$ & 71.0 \\
$\lambda_{\text {bdfim.dt }}$ & 0.84 & $\mu_{\text {bdfim.dt }}$ & 65.8 \\
\hline
\end{tabular}

The reliability model parameters, based on equations (5) to (34) and data from Tables IV and V are given in Table VI. The improved availability and reliability of the BDFIM compared to 
the conventional DFIG is due to the absence of the brush gear. The improvement in gearbox reliability for the case of two-stage $(2 \mathrm{~g})$ compared to the three-stage $(3 \mathrm{~g})$ gearbox is due to the absence of the high speed parallel gear stage. The above two reliability improvements have resulted in the overall $13.5 \%$ reduction in the failure rate and $7.3 \%$ reduction in the repair rate of the drivetrain when high-speed DFIG drivetrain is replaced by the medium-speed BDFIM drivetrain.

\section{ACKNOWLEDGMENT}

The research has partly been funded by Innovate UK (project no: 56639) and in collaboration with Green Solution Engineering (GSE) Limited, Cambridge, UK.

\section{REFERENCES}

[1] Global Wind Energy Council., 'Global Wind Report 2018' (GWEC, 2019), pp. 22-30.

[2] Z. Zhang, A. Matveev, R. Nilssen, A. Nysveen, 'Ironless PermanentMagnet Generators for Offshore Wind Turbines', IEEE Transactions on Industry Applications, Vol. 50, pp. 1835-1846, June 2014.

[3] A. Oraee, R. McMahon, E. Abdi, S. Abdi, S. Ademi, 'Influence of polepair combinations on the characteristics of the brushless doubly fed induction generator', IEEE Transactions on Energy Conversion, Vol. 35, pp. 1151-1159, September 2020.

[4] E. Abdi, R. McMahon, P. Malliband, S. Shao, M. Mathekga, P. Tavner, S. Abdi, A. Oraee, T. Long, and M. Tatlow, 'Performance analysis and testing of a $250 \mathrm{kw}$ medium-speed brushless doubly fed induction generator', IET Renewable Power Generation, Vol. 7, No. 6, pp. 631638, 2013.

[5] H. Arabian, H. Oraee, P. Tavner, 'Failure modes and effects analysis (FMEA) for wind turbines', International Journal of Electrical Power \& Energy Systems, Vol. 32, No. 7, pp. 817-824, 2010.

[6] M. Afshar, S. Abdi, M. Ebrahimi, S. Mortazavizadeh, 'Static Eccentricity Fault Detection in Brushless Doubly-Fed Induction Machines based on Motor Current Signature Analysis', 45th annual conference of the IEEE industrial electronics society, pp. 1369-1374, Lisbon, October 2019.

[7] M. Afshar, A. Tabesh, M. Ebrahimi, S. Khajehoddin, 'Stator short circuit fault detection and location methods for brushless DFIMs using nestedloop rotor slot harmonics', IEEE Transactions on Power Electronics, Vol. 35, pp. 8559-8568, August 2020.

[8] T. Logan, R. McMahon, K. Seffen, 'Noise and vibration in brushless doubly fed machine and brushless doubly fed reluctance machine', IET Electric Power Applications, vol. 7, pp. 1-10, 2014.
[9] S. Abdi, E. Abdi, R. McMahon, 'A Study of Unbalanced Magnetic Pull in Brushless Doubly Fed Machines', IEEE Transactions on Energy Conversion, vol. 30, pp. 1218-1227, 2015.

[10] P. Tavner, A. Higgins, H. Arabian, H. Long, Y. Feng, 'Using an FMEA method to compare prospective wind turbine design reliabilities', European Wind Energy Conference, Technical Track, EWEA, Poland 2010.

[11] S. Abdi, E. Abdi, H. Toshani, R. McMahon, 'Vibration Analysis of Brushless Doubly Fed Machines in the Presence of Rotor Eccentricity', IEEE Transactions on Energy Conversion, Vol. 35, pp. 1372-1380, September 2020.

[12] S. Abdi, E. Abdi, A. Oraee and R. McMahon, "Equivalent circuit parameters for large brushless doubly fed machines (bdfms)," IEEE Transactions on Energy Conversion, Vol. 29, No. 3, pp. 706-714, 2014.

[13] R. Carlson, H. Voltolini, F. Runcos, P. Kuo-Peng, and N. Baristela, 'Performance analysis with power factor compensation of a $75 \mathrm{kw}$ brushless doubly fed induction generator prototype', IEEE International Conference on Electric Machines and Drives, 2010.

[14] H. Liu and L. Xu, 'Design and performance analysis of a doubly excited brushless machine for wind power generator application', IEEE International Symposium on Power Electronics for Distributed Generation Systems, pp. 597-601, 2010.

[15] T. Long, S. Shao, P. Malliband, E. Abdi, R. A. McMahon, 'Crowbarless Fault Ride-Through of the Brushless Doubly Fed Induction Generator in a Wind Turbine Under Symmetrical Voltage Dips', IEEE Transactions on Industrial Electronics, Vol. 60, pp. 2833-2841, July 2013.

[16] S. Abdi, E. Abdi, A, Oraee, R. McMahon, 'Optimisation of magnetic circuit for brushless doubly fed machines', IEEE Transactions on Energy Conversion, Vol. 30, No, 4, pp. 1611-1620, Secember 2015.

[17] R. Sadeghi, S. Madani, M. Kashkooli, 'Grid synchronization of brushless Doubly Fed Induction Generator using direct torque control', IEEE power electronics and drive systems Technologies Conference, pp. 53-57, Tehran, 2016.

[18] M. Wilkinson, B. Hendriks, F. Spinato, E. Gomez, H. Bulacio, J. Roca, P. Tavner, Y. Feng, and H. Long, 'Methodology and results of the reliawind reliability field study', European Wind Energy Conference (EWEC), 2010.

[19] H. Arabian-Hoseynabadi, H. Oraee, P. Tavner, 'Wind turbine productivity considering electrical subassembly reliability', Renewable Energy, Vol. 35, pp. 190-197, 2010.

[20] K. Smolders, H. Long, Y. Feng, P. Tavner, 'Reliability analysis and prediction of wind turbine gearboxes', in European Wind Energy Conference, Scientific Track, pp. 2660-2670, Poland, 2010 